\title{
Recurrent sinusitis and periorbital cellulitis secondary to congenital zuckerkandl dehiscence
}

\begin{abstract}
Rhinosinusitis is the most common cause of orbital infections in children. Its orbital complications are rare, dangerous and their incidence increases in children. Its most common complications of RS, in order of increasing severity, in children: orbital cellulitis, subperiosteal abscess, intraorbital abscess and cavernous sinus thrombosis. The spread of infectious agents from the ethmoid sinuses to the periosteum of the orbit may occur directly through congenital/ acquired dehiscences of the lamina papyracea, or via haematogenous spread through the valveless ophthalmic venous channels. Congenital, traumatic, or iatrogenic defects of the lamina papyracea or simple anatomical variation can cause dehiscences (weakness) of the lamina papyracea. Therefore, the physicians and immunologists should be aware of uncommon anatomic variations causing recurrent paranasal sinusitis such as congenital dehiscence of lamina papyracea before suspecting of primary immune deficiency.
\end{abstract}

Keywords: zuckerkandl dehiscence, lamina papyracea; recurrent sinusitis, periorbital cellulitis
Volume I Issue 5 - 2014

Oner Ozdemir, Bahri Elmas

Department of Pediatrics, Sakarya University, Turkey

\begin{abstract}
Correspondence: Oner Ozdemir, Division of Allergy and Immunology, Department of Pediatrics, Research and Training Hospital of Sakarya University, Faculty of Medicine, Sakarya University, Adnan Menderes Cad, Sa $\square$ lık Sok. No: 195, Adapazarı, Sakarya, Turkey, Tel 90-264-4445400, Fax 90-264 -275 91 92, Email onerozdemir@sakarya.edu.tr
\end{abstract}

Received: November 0I, 2014 | Published: November 19, 2014
Abbreviations: RS, rhinosinusitis; FESS, functional endoscopic sinus surgery; PID, primary immunodeficiency diseases

\section{Introduction}

Rhinosinusitis (RS) is the most common cause of orbital infections in children. Although the incidence of RS complications has decreased since the launch of antibiotics, the risk of orbital involvement remains important. Orbital complications of RS are rare, dangerous and their incidence increases in children. They can result in permanent blindness or death if not treated immediately. The most common complications of RS, in order of increasing severity, in children: orbital cellulites, subperiosteal abscess, intraorbital abscess and cavernous sinus thrombosis. ${ }^{1}$ In a recent original article by Al-Madani et al reported the prevalence of orbital complications among children and adults with acute RS. They evaluated 616 patients attending ENT clinic with sinusitis from January 2010 until January 2012. Orbital complications were seen in 36 patients (5.8\%). Among 36 patients; 26 patients $(72.2 \%)$ were children (21 had preseptal and 5 had orbital cellulitis) and ten patients $(27.8 \%)$ were adults ( 5 with preseptal, 3 with orbital cellulitis and 2 with abscess). The most common orbital complication was preseptal cellulitis (72.2\%) followed by orbital cellulitis $(22.2 \%)$ and abscess $(5.6 \%)$. Orbital complications of sinusitis are commoner in children than adults and have favorable prognosis. ${ }^{2}$

Orbital complications of pediatric RS spread from the ethmoid sinus to the subperiosteal space underlying the lamina papyracea. The lamina papyracea can be defined as the wafer-thin barrier between the ethmoid sinuses and the orbit. ${ }^{3}$ The spread of infectious agents from the ethmoid sinuses to the periosteum of the orbit may occur directly through congenital/acquired dehiscences of the lamina papyracea, or via haematogenous spread through the valveless ophthalmic venous channels.

Orbital complications of pediatric RS spread from the ethmoid sinus to the subperiosteal space underlying the lamina papyracea. The lamina papyracea can be defined as the wafer-thin barrier between the ethmoid sinuses and the orbit. ${ }^{3}$ The spread of infectious agents from the ethmoid sinuses to the periosteum of the orbit may occur directly through congenital/acquired dehiscences of the lamina papyracea, or via haematogenous spread through the valveless ophthalmic venous channels. Rare anatomic variations such as Zuckerkandl (the lamina papyracea) dehiscences are encountered in patients with chronic paranasal sinusitis who have undergone functional endoscopic sinus surgery (FESS). Bony dehiscences are readily demonstrable in CT scans of the paranasal sinuses. Serial CT scan images conducted in 100 chronic paranasal sinusitis patients showed nontraumatic protrusion of orbital contents through dehiscence of lamina papyracea in $1 \%$ of patients. ${ }^{4} \mathrm{RS}$ orbital complications are usually unilateral and probably related to asymmetries in the anatomy of the lamina papyracea on the infected and noninfected sides. Congenital dehiscences of the lamina papyracea are distributed asymmetrically. Acquired dehiscences are usually asymmetrical as well, since they are caused by trauma that rarely affects both sides of the face equally. These asymmetries would favor the spread of disease to the one orbit.

There are several warning signs of primary immunodeficiency diseases (PID) to predict earliest possible diagnosis. One of them is two or more new sinus infections within 1 year in the absence of allergy [5]. Since dehiscence of the left lamina papyracea may cause recurrent sinus and orbital infections, it is confused with PID and should be differentiated from it in clinic. Consequently, to learn and be aware of dehiscences of the lamina papyracea is very important to physicians dealing with recurrent paranasal sinusitis and periorbital infections.

\section{Aim}

Here, we describe a patient with congenital dehiscence of the left lamina papyracea causing recurrent sinusitis and periorbital cellulitis.

\section{Case report}

A 7-year-old male with three episodes of both preseptal / periorbital cellulitis and left maxillary sinusitis are presented here. He was hospitalized two times in the last year due to left-sided periorbital 
swellings and treated with intravenous antibiotics and sent home on oral cephalosporins. He then remained well until 2 months ago, when he developed a similar episode, although this one was caught earlier and treated with intramuscular and oral antibiotics and did not require hospitalization. In his review of systems: he has not had any other serious infections besides these two episodes of periorbital cellulitis. Family history was negative for allergic diseases and immunodeficiencies. Environmental history: The home environment is a rural house with an outdoor pet dog and no smokers. Physical examination showed that he is a well-developed, well-nourished child who appears healthy and in no acute distress. The remainder of the physical exam is unremarkable. Laboratory investigations: The first two sinus CT scans showed a chronically inflamed left lacrimal sac and periorbital cellulitis. Both maxillary sinuses had significant mucosal thickening, although there were no air-fluid levels. Interestingly, the last sinus CT with contrast showed two areas of congenital dehiscence of left lamina papyracea (Figure 1a $\& 1 b)$. The immunoglobulin and IgG subgroup levels were normal and he had excellent antibody titers against most of the pneumococcal serotypes. Skin tests showed negative reactions on both prick and intradermal test to all of the common aeroallergens.

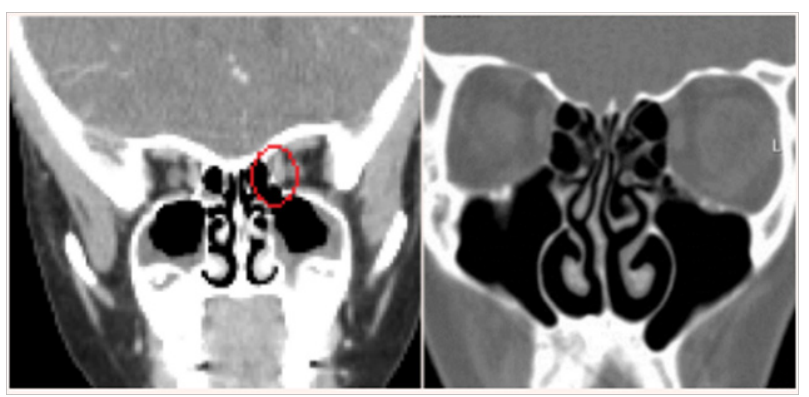

Figure I a \& b Normal sinus CT is shown on the right figure. On the left, sinus CT with contrast showed two areas of congenital dehiscence of left lamina papyracea.

\section{Discussion}

Congenital, traumatic, or iatrogenic defects of the lamina papyracea or simple anatomical variation can cause dehiscences (weakness) of the lamina papyracea. ${ }^{6}$ Age-related etiologic factors have also been suggested. For instance: trauma to the lamina papyracea during drilling of the mastoid during cochlear implantation and intranasal ethmoidectomy, up to $10 \%$.

The exact prevalence of Zuckerkandl dehiscences in adult or children is not known. Zuckerkandl dehiscences is usually an incidental finding in $0.8 \%(6 / 783)$ of the patients $\mathrm{CT}$ scans taken to detect polyps or assess chronic paranasal sinusitis. ${ }^{4} \mathrm{CT}$ scans in $2 / 400(0.5 \%)$ showed dehiscence of the lamina papyracea in patients undergoing FESS and particularly evaluating anatomic variations. ${ }^{3}$ The severity may be greatly variable, with a small defect to more than $2 / 3$ of the lamina papyracea involved. In a recent study, 1.024 sinus $\mathrm{CT}$ of patients with subjective sinonasal symptoms were evaluated. The aim was to analyze the incidence and CT features of nontraumatic prolapse of the orbital content into the ethmoid sinus. Ethmoidal prolapse of the orbital content was found in 67 out of 1024 patients (6.5\%). The incidence during childhood was significantly low (1.9\%) as compared with that of adult. There was a tendency of increasing incidence with older age. Only four patients $(0.4 \%)$ had bilateral lesions. The higher incidence in adult may suggest age-related acquired etiologic factors besides congenital dehiscence or weakness of the lamina papyracea. ${ }^{8}$

While many types of PID have been identified, all types share similar symptoms-notably recurring, frequent infections that can be long-lasting and severe. To diagnose PID in a patient, secondary causes for recurrent infections such as Zuckerkandl dehiscences should be ruled out. Secondary factors leading frequent and recurrent infections more common than a PID. And surely, it is not solely sufficient as one out of 10 warning signs defined by the Jeffrey Modell Foundation for suspecting PID to make an absolute conclusion about diagnosing PID. ${ }^{5}$

\section{Conclusion}

Therefore, the physicians and immunologists should be aware of uncommon anatomic variations causing recurrent paranasal sinusitis such as congenital dehiscence of left lamina papyracea before suspecting of PID.

\section{Acknowledgements}

None.

\section{Conflicts of Interest}

There is no conflict of interest.

\section{Funding}

None.

\section{References}

1. Siedek V, Kremer A, Betz CS, et al. Management of orbital complications due to rhinosinusitis. Eur Arch Otorhinolaryngol. 2010;267(12):18811886.

2. Al-Madani MV, Khatatbeh AE, Rawashdeh RZ, et al. The prevalence of orbital complications among children and adults with acute rhinosinusitis. Braz J Otorhinolaryngol. 2013;79(6):716-719.

3. Reid JR. Complications of pediatric paranasal sinusitis. PediatrRadiol. 2004;34(12):933-942.

4. Moulin G, Dessi P, Chagnaud C, et al. Dehiscence of the lamina papyracea of the ethmoid bone: CT findings. AJNR Am J Neuroradiol. 1994;15(1):151-153.

5. ht tps://s 3 . a mazon aw s. com/j m f $-\mathrm{stage/pdfs/}$ General10WarningSignsFINAL.pdf

6. Meyers RM, Valvassori G. Interpretation of anatomic variations of computed tomography scans of the sinuses: a surgeon's perspective. Laryngoscope. 1998;108(3):422-425.

7. Hoffman RA, Parisier SC, Roland JT Jr. In reference to Orbital sequelae of rhinosinusitis after cochlear implantation in children. Laryngoscope. 2007;117(8):1505-1506

8. Han MH, Chang KH, Min YG, et al. Nontraumatic prolapse of the orbital contents into the ethmoid sinus: evaluation with screening sinus CT. Am J Otolaryngol. 1996;17(3):184-189. 